\title{
TI.35.1
}

\section{Baseline Expectations: Community Dispute Resolution Process}

(superseded by TI.105.1 and then TI.105.2)

- PDF: CommunityDisputeResolutionProcess.pdf

- Text: CommunityDisputeResolutionProcess.txt

\section{More Information}

\begin{tabular}{|l|l|}
\hline Repository ID & TI.35.1 \\
\hline Persistent URL & http://doi.org/10.26869/TI.35.1 \\
\hline Title & Baseline Expectations: Community Dispute Resolution Process \\
\hline Authors & Tom Barton and members of the InCommon CTAB \\
\hline Sponsor & InCommon Community Trust and Assurance Board (CTAB) \\
\hline Review & \\
\hline Status & Preserve \\
\hline Publish Date & September 14, 2017 \\
\hline DOI & $10.26869 / T I .35 .1$ \\
\hline Signature & \\
\hline Deprecated & Yes \\
\hline Future Review & \\
\hline Supersedes & \\
\hline Format & PDF, Text \\
\hline Related Docs & \\
\hline Development Location & CommunityDisputeResolutionProcess.docx \\
\hline IP Framework & CC-BY 4.0 \\
\hline Subject Tags & incommon, federation, assurance, trust, framework \\
\hline Notes & \\
\hline & \\
\hline
\end{tabular}

